\title{
Metamaterial tuning by manipulation of near-field interaction
}

\author{
David A. Powell, ${ }^{1, *}$ Mikhail Lapine, ${ }^{2,1}$ Maxim V. Gorkunov, ${ }^{3}$ Ilya V. Shadrivov, ${ }^{1}$ and Yuri S. Kivshar ${ }^{1}$ \\ ${ }^{1}$ Nonlinear Physics Centre, Research School of Physics and Engineering, Australian National University, Canberra, \\ Australian Capital Territory 0200, Australia \\ ${ }^{2}$ Department Electronics and Electromagnetics, Faculty of Physics, University of Seville, Avda. Reina Mercedes s/n, 41015 Seville, Spain \\ ${ }^{3}$ A. V. Shubnikov Institute of Crystallography, Russian Academy of Sciences, Lenin Ave. 59, 119333 Moscow, Russia
}

(Received 6 December 2009; revised manuscript received 14 June 2010; published 19 October 2010)

\begin{abstract}
We analyze the near-field interaction between the resonant subwavelength elements of a metamaterial and present a method to calculate the electric and magnetic interaction coefficients. We show that by adjusting the relative configuration of the neighboring split ring resonators it becomes possible to manipulate this near-field interaction, and thus tune the response of metamaterials. We use the results of this analysis to explain the experimentally observed tuning of microwave metamaterials.
\end{abstract}

DOI: 10.1103/PhysRevB.82.155128

PACS number(s): 41.20.Jb, 78.70.Gq, 42.70.Qs

\section{INTRODUCTION}

Metamaterials, which are typically regular arrays of subwavelength resonant particles, offer us a new degree of freedom in controlling the electromagnetic response of matter. Thus we are no longer completely constrained by the properties of existing materials but can tailor the response in an almost arbitrary fashion, for example, achieving very high, ${ }^{1}$ very low, ${ }^{2}$ and negative ${ }^{3}$ values of refractive index, permittivity and/or permeability. Because of the inherently strong dispersion of resonant metamaterials, they must be modified in order to operate in a different frequency band. Therefore, there is a significant push to have a further degree of control over these materials - tunability of their response.

Fortunately, the engineered nature of metamaterials allows their properties to be controlled externally, either by dynamically modifying their structure or by adding some nonlinear inclusion and controlling with external fields. ${ }^{4}$ Examples of the latter approach include the introduction of varactor diodes, ${ }^{5}$ ferroelectrics ${ }^{6}$ and photoconductive semiconductors. ${ }^{7}$ On the other hand, even without resorting to such exotic (and often lossy) constituents, there is a great deal of freedom to manipulate the structure itself, and this is the approach we take here. We consider specifically the split ring resonator (SRR) as one of the most important metamaterial elements, noting that while the fine details of near-field interaction are structurally specific, our approach can be applied to a wide variety of structures.

An analytical model for the magnetic response of a subwavelength array of identically oriented wire loops loaded with a capacitance ${ }^{8}$ takes into account the mutual interaction of all the elements in the lattice, which is essential for deriving the effective permeability correctly. Although that analysis is limited to the quasistatic case accounting only for magnetic near-field interactions, it is crucial for revealing the consequence of lattice changes. These tend to be overlooked by otherwise rigorous approaches which include spatial dispersion but develop Lorentz local field approaches, based on nearest-neighbor interaction ${ }^{9}$ or point-dipole approximation. $^{10}$

In particular, it was pointed out in Ref. 8 that the resonant frequency of the metamaterial permeability can be altered by varying the lattice constants without changing the structural units. This scheme is illustrated in Fig. 1(a) and has been verified by experiments in Ref. 11. However, a practical consequence of this change in lattice constant is that the sample size also changes correspondingly. More recently, an alternative approach was suggested in Ref. 12: introducing a shift between layers in order to create a monoclinic lattice with the shift increasing linearly between layers, as shown in Fig. 1(b). This configuration keeps the density of elements within the metamaterial constant and can tune the coupling between neighboring particles to modify the response of the complete metamaterial. However, for finite-size samples, this shift inevitably results in a significant change in sample shape. Thus, for practical purposes, we have proposed a superlattice type of geometry, whereby only every second layer is shifted by the same amount, as shown in Fig. 1(c). This tuning scheme proved to be robust and allows significant manipulation of the resonant frequency with only a small change in the sample geometry. ${ }^{12}$ Therefore, the sample geometry and its effective properties can be engineered almost independently to achieve the desired manipulation of electromagnetic waves.

However, as we demonstrate below, this structural tuning of metamaterials depends very strongly on the nature of the near-field interactions. Since metamaterial elements such as split ring resonators are usually not highly symmetric, the relative orientation of particles within the lattice is of key importance. This effect is not described by existing circuit theory models and can give rise to some surprising experimental results, which we present here.
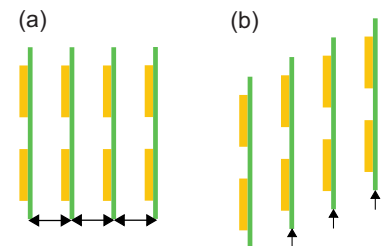

(c)

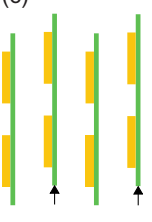

FIG. 1. (Color online) Several approaches to modify the lattice for metamaterial tunability: (a) a change in the lattice constant (Ref. 11), (b) a continuous shift of the layers (Ref. 12), and (c) a superlattice of alternating shifts of layers (Ref. 12). 
In order to understand the coupling mechanisms and how they are affected by the lattice shift, it is useful to start with the simplest geometry-a pair of split ring resonators. Several authors have conducted numerical and experimental investigations of coupling between metamaterial elements for different types of elements and relative orientation between them, in microwave ${ }^{13,14}$ and optical frequency ranges. (See the overview in Ref. 15 and references therein, as well as Refs. 16-21.) A detailed study has previously been undertaken on tailoring the geometric arrangement of a pair of coupled one-dimensional SRR arrays to engineer the dispersion curves of magnetoinductive waves. ${ }^{22}$

As we have shown recently, ${ }^{12}$ a rough qualitative understanding of the structural tuning can be achieved by using circuit theory with purely inductive coupling between SRRs. However, this approximation fails to provide a quantitative understanding of the metamaterial tuning in question and, most importantly, does not explain the observed strong influence of the relative SRR orientation. Therefore, below we develop a distinct model based on the calculation of the fundamental mode of a single resonator. The knowledge of the current and charge distributions within the mode allows calculating the coupling constants of a pair of split rings. These constants are then used to explain the experimentally observed tuning response of our metamaterial samples.

In Sec. II, we develop our approach to calculate the metamaterial coupling, including a discussion of the limitations of other purely analytical methods. In Sec. III, we apply our approach to the study of interaction between a pair of split ring resonators which are shifted laterally relative to each other and explain quantitatively how the shift affects the position of the fundamental resonance. In Sec. IV, we apply these results to a bulk metamaterial and identify the mechanisms at work in the experimental tuning of a metamaterial slab in a waveguide. Finally, Sec. V concludes the paper with further discussions and outlook.

\section{NEAR-FIELD INTERACTION IN METAMATERIALS}

Considering a single SRR, it is known ${ }^{13}$ that it possesses a discrete set of eigenmodes (standing waves) with corresponding eigenfrequencies. In an arbitrarily excited SRR, the currents and charges can be represented as a superposition of the eigenmodes. The fundamental mode with the lowest frequency is relevant for the magnetic resonance in SRRs. On the frequency scale this mode is well isolated from the higher-order modes, and we can restrict ourselves to the single-mode approximation neglecting the excitation of higher modes.

The time-dependent charge density $\rho$ and current density $\mathbf{J}$ in a resonant element with excited fundamental mode can be written in the most general form of a standing wave

$$
\begin{gathered}
\rho(\mathbf{x}, t)=Q(t) q(\mathbf{x}), \\
\mathbf{J}(\mathbf{x}, t)=I(t) \mathbf{j}(\mathbf{x}),
\end{gathered}
$$

where $q$ and $\mathbf{J}$ describe the charge and current distributions in space. In SRRs, the variation in the current distribution across the width of the conductive track could be neglected,
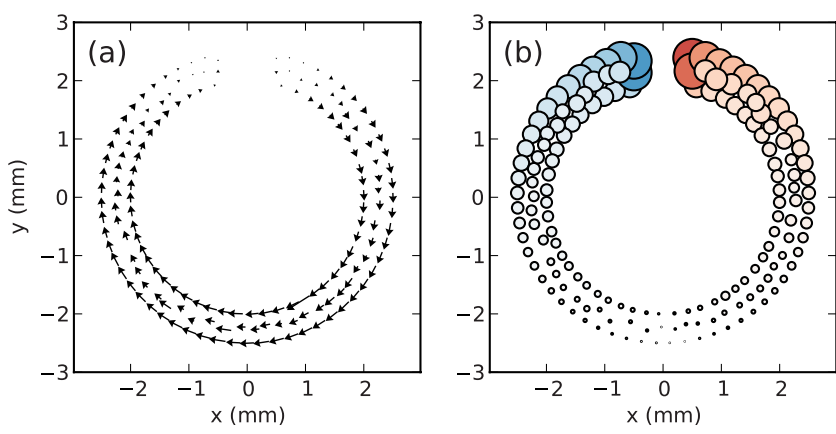

FIG. 2. (Color online) Numerically calculated (a) current and (b) charge distribution across an SRR at resonance.

however, for generality our approach takes into account the complete three-dimensional surface-current distribution.

To satisfy the conservation of charge

$$
\nabla \cdot \mathbf{J}=-\frac{\partial \rho(\mathbf{x}, t)}{\partial t}
$$

we imply that

$$
\begin{gathered}
I(t)=\dot{Q}(t), \\
\nabla \cdot \mathbf{j}(\mathbf{x})=-q(\mathbf{x}) .
\end{gathered}
$$

Thus if the current is known, it is easy to find the charge distribution and vice versa. The mode profile obtained numerically for our SRR geometry is shown in Fig. 2. The current $\mathbf{j}$ is symmetric and reaches its maximum at the point opposite to the gap. In accordance with Eq. (5), the charge distribution $q(\mathbf{x})$ is antisymmetric and goes through zero where $\mathbf{j}(\mathbf{x})$ is maximal. We see that $q(\mathbf{x})$ reaches its maximum magnitude near the gap.

In the single-mode approximation, the dynamics of the SRR can be fully described by the time-dependent amplitude $Q(t)$, and we may write the SRR Lagrangian as a sum of terms quadratic in $Q$ and $\dot{Q}$

$$
\mathcal{L}=A \dot{Q}^{2}-B Q^{2},
$$

where $A$ and $B$ are constants which will be discussed below. Accordingly, the SRR energy reads

$$
E=\dot{Q} \frac{\partial \mathcal{L}}{\partial \dot{Q}}-\mathcal{L}=A I^{2}+B Q^{2}
$$

and is nicely separated into inductive (magnetic) and capacitive (electric) parts. Clearly, for a passive SRR we require $A \geq 0, B \geq 0$.

The Lagrangian equation of motion

$$
\frac{d}{d t} \frac{\partial \mathcal{L}}{\partial \dot{Q}}=\frac{\partial \mathcal{L}}{\partial Q}
$$

yields that the dynamics of a single SRR is described by the oscillator equation for the charge amplitude 


$$
\ddot{Q}(t)+\omega_{0}^{2} Q(t)=0,
$$

and the fundamental mode resonance occurs at the frequency $\omega_{0}=\sqrt{B / A}$.

Note that in contrast to the known modifications of the Lagrangian formalism for metamaterials (see, e.g., Ref. 23), here we do not rely on an equivalent circuit model. Although one might identify the parameters $2 A$ and $1 / 2 B$ as effective inductance and capacitance, respectively, below we evaluate them explicitly from the known fundamental mode shape. In fact, the strongly inhomogeneous mode profile (see Fig. 2) suggests that it is unlikely that the correct values of the parameters would agree with those calculated from a circuit analysis. Additionally, to find the resonant frequency, we do not need to calculate $A$ and $B$ explicitly, only their ratio.

For the purposes of our analysis, it is sufficient to consider the case of a pair of SRRs, and it will subsequently be shown that this explains all of the important features observed in our experiments with a bulk metamaterial. In this case, the Lagrangian can be written as a sum of the single SRR Lagrangians and coupling terms, which we also write as quadratic in currents and charges

$$
\mathcal{L}=A\left(\dot{Q}_{1}^{2}+\dot{Q}_{2}^{2}+2 \alpha \dot{Q}_{1} \dot{Q}_{2}\right)-B\left(Q_{1}^{2}+Q_{2}^{2}+2 \beta Q_{1} Q_{2}\right) .
$$

The parameters $\alpha$ and $\beta$ are the dimensionless constants of magnetic and electric near-field interaction, respectively.

The corresponding Lagrangian equations of motion yield the system of ODEs for the time-dependent amplitudes $Q_{1,2}$

$$
\begin{aligned}
& \ddot{Q}_{1}+\omega_{0}^{2} Q_{1}=-\alpha \ddot{Q}_{2}-\beta \omega_{0}^{2} Q_{2}, \\
& \ddot{Q}_{2}+\omega_{0}^{2} Q_{2}=-\alpha \ddot{Q}_{1}-\beta \omega_{0}^{2} Q_{1} .
\end{aligned}
$$

Solving these equations one finds that a pair of resonators exhibits two resonances: symmetric and antisymmetric. For the symmetric resonance, $Q_{1}=Q_{2}$, which yields the resonant frequency

$$
\omega_{S}=\omega_{0} \sqrt{\frac{1+\beta}{1+\alpha}}
$$

while the antisymmetric mode with $Q_{1}=-Q_{2}$ has the frequency

$$
\omega_{A S}=\omega_{0} \sqrt{\frac{1-\beta}{1-\alpha}} .
$$

The described resonance splitting is well known in the theory of harmonic oscillators. Generally, bringing together two oscillators of the same resonant frequency introduces coupling between them, which results in splitting into two modes. Examples have been shown of SRR resonant frequency as a function of some coupling parameter, e.g., mutual orientation ${ }^{24}$ or twist angle, ${ }^{25}$ and typically demonstrate a splitting or hybridization of modes.

As we see, the direction and strength of the resonance shift are determined by the coupling constants $\alpha$ and $\beta$. To evaluate them, we use the expression for the electromagnetic energy following from Lagrangian (10):

$$
E=A\left(I_{1}^{2}+I_{2}^{2}+2 \alpha I_{1} I_{2}\right)+B\left(Q_{1}^{2}+Q_{2}^{2}+2 \beta Q_{1} Q_{2}\right) .
$$

The first group of terms gives the magnetic energy and the second group describes the electric energy.

A possible route to calculate $\alpha$ and $\beta$ is to approximate the electric and magnetic response of each ring by a few terms of the multipole expansion. The problem with this approach is that it is based on the assumption that the observer (i.e., the second SRR) is at a large distance compared to the dimensions of the source. This requirement is strongly violated in our metamaterial samples, where the separation between rings is actually much smaller than the outer ring diameter. This is essential for achieving strong tuning by lattice manipulation.

Therefore we have chosen to calculate $\alpha$ and $\beta$ numerically from the known charge and current distributions, $q(\mathbf{x})$ and $\mathbf{j}(\mathbf{x})$, of the fundamental mode in a single SRR. Indeed, in the single-mode approximation, the energy of a pair of SRRs reads

$$
\begin{aligned}
E= & Q_{1}^{2} W_{E, 11}+Q_{2}^{2} W_{E, 22}+2 Q_{1} Q_{2} W_{E, 12}+I_{1}^{2} W_{H, 11}+I_{2}^{2} W_{H, 22} \\
& +2 I_{1} I_{2} W_{H, 12},
\end{aligned}
$$

where the parameters

$$
\begin{gathered}
W_{E, m n}=\int_{V_{m}} d^{3} x \int_{V_{n}} d^{3} x^{\prime} \frac{q(\mathbf{x}) q\left(\mathbf{x}^{\prime}\right)}{4 \pi \epsilon_{0}\left|\mathbf{x}-\mathbf{x}^{\prime}\right|}, \\
W_{H, m n}=\int_{V_{m}} d^{3} x \int_{V_{n}} d^{3} x^{\prime} \frac{\mu_{0} \mathbf{j}(\mathbf{x}) \cdot \mathbf{j}\left(\mathbf{x}^{\prime}\right)}{4 \pi\left|\mathbf{x}-\mathbf{x}^{\prime}\right|} .
\end{gathered}
$$

The integrals can be easily evaluated once the charge and current distributions are known. The integrations over $\mathbf{x}$ and $\mathbf{x}^{\prime}$ are over the same ring if $m=n$ or over different rings otherwise. Accordingly, $V_{1}$ is a volume containing only the first ring and $V_{2}$ is a volume containing only the second. The singular terms at $x=x^{\prime}$ are handled using the analytical formulas given in Ref. 26.

Comparing Eqs. (15) and (16) shows that the coupling parameters can be evaluated as

$$
\alpha=\frac{W_{H, 12}}{W_{H, 11}}, \quad \beta=\frac{W_{E, 12}}{W_{E, 11}} .
$$

For comparison purposes, when inductive coupling is the dominant interaction mechanism between the SRRs in a metamaterial, we are able to consider an array of split rings as an array of current loops with some mutual inductance between them. This approach can then be used to define the effective permeability of a metamaterial sample. ${ }^{8}$ In particular, for thin wire loops with their axes oriented in the same direction, the mutual inductance can be found ${ }^{27}$ by numerical integration

$$
\begin{aligned}
L_{n n^{\prime}}(\mathbf{r})= & \frac{\mu_{0} r_{0}^{2}}{4 \pi} \int_{0}^{2 \pi} \int_{0}^{2 \pi} d \varphi_{1} d \varphi_{2} \cos \left(\varphi_{1}-\varphi_{2}\right) \\
& \times\left\{\rho^{2}+z^{2}+2 r_{0}^{2}\left[1-\cos \left(\varphi_{1}-\varphi_{2}\right)\right]\right. \\
& \left.+2 \rho r_{0}\left(\cos \varphi_{2}-\cos \varphi_{1}\right)\right\}^{-1 / 2},
\end{aligned}
$$

where the distance vector $\mathbf{r}$ between the ring centers has 


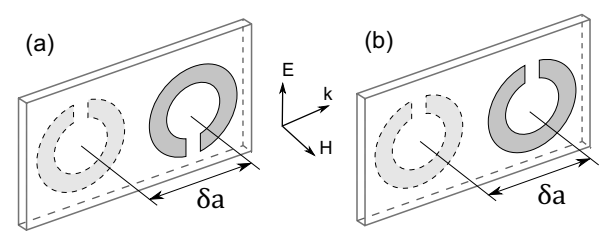

FIG. 3. Geometry of the pair of rings (a) broadside-coupled and (b) gap-to-gap orientation.

been decomposed into a radial component $\rho$ and axial component $z, r_{0}$ is the ring radius, and $\varphi_{1}$ and $\varphi_{2}$ represent the angle about each ring. We can use the calculated mutual inductance to define an equivalent magnetic interaction parameter

$$
\alpha_{L}=L_{n n^{\prime}} / L
$$

(where $L$ denotes the self-inductance of one element) which should approximate the interaction energy calculated from Eq. (19). Asymptotically this interaction decays as $1 / r$ so in a large array the nearest neighbors provide the strongest contribution but do not necessarily dominate over all others. Clearly, this interaction is highly anisotropic, ${ }^{22}$ being positive for rings on the same axis, but negative for rings in the same plane.

It is also possible to develop equivalent circuit models to calculate the electric interaction between rings. For a pair of coaxial rings, the total interaction can be reliably modeled as a circular parallel conductor transmission line, as for broadside-coupled (bc) split ring resonators ${ }^{28}$ or, alternatively, with an extended circuit model accounting for the distributed capacitance and inductance. ${ }^{29}$ However, once we introduce some offset between the rings, these approaches are not applicable and so we do not consider them here.

\section{TUNING INTERACTION BETWEEN A PAIR OF SPLIT RING RESONATORS}

Having developed an approach for calculating near-field interaction between a pair of rings, we now apply it to a canonical system which has the basic properties of our experimental arrangement. We consider a pair of SRRs, either identically [gap-to-gap (gtg)] oriented or rotated by $180^{\circ}$ with respect to each other [broadside-coupled (bc)] and subject to a lateral offset $\delta a$. The geometry and incident polarization are shown in Fig. 3. The rings have average radius $r_{0}=2.25 \mathrm{~mm}$, track width of $0.5 \mathrm{~mm}$, metal thickness of 0.03 $\mathrm{mm}$, gap width of $1 \mathrm{~mm}$, and are separated in the transverse direction by $1.5 \mathrm{~mm}$. The resulting resonant frequency is $10.6 \mathrm{GHz}$.

We plot the interaction energy calculated from Eq. (19) in Fig. 4 for different offsets between the rings. It can be seen that the electric coupling parameter $\beta$ is nearly symmetric between the two configurations. This can be understood from Fig. 2, where we see that the charge distribution has a strong dipole component oriented in the $x$ direction. For the symmetric mode of the broadside-coupled orientation the charges accumulated on the closest sides of the SRRs have opposite signs, the total-charge distribution has the nature of a pair of

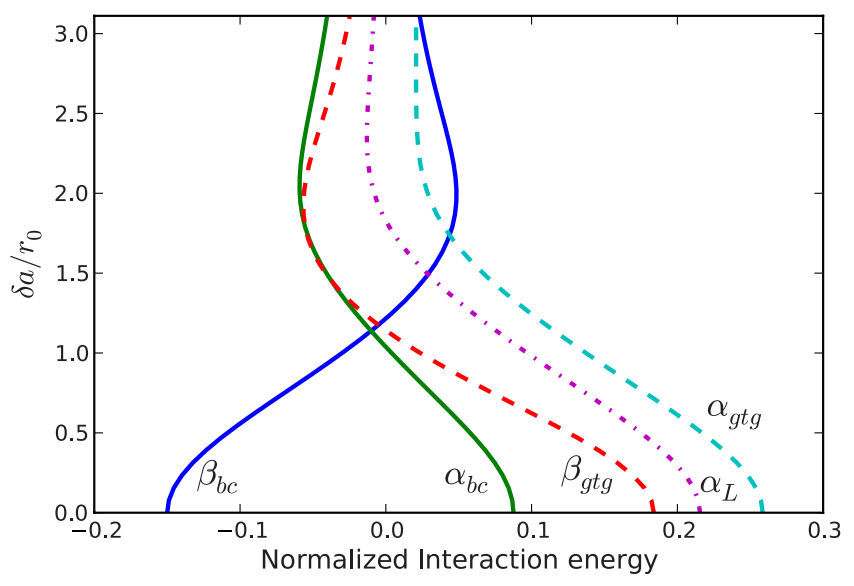

FIG. 4. (Color online) Magnetic $(\alpha)$ and electric $(\beta)$ coupling parameters for broadside coupled (bc) and gap-to-gap (gtg) orientation of a pair of shifted SRRs. The magnetic interaction calculated from the mutual inductance is given as $\alpha_{L}$.

antiparallel dipoles, thus $W_{E, 12}<0$, and $\beta$ is also negative. In contrast, in the gap-to-gap orientation, the closest charges are of the same sign, the total-charge distribution becomes like a pair of parallel dipoles and the parameter $\beta>0$.

At an offset of about one ring radius $\left(\delta a \approx r_{0}\right)$, the charge on one ring is approximately equidistant from both positive and negative charges on the opposite ring, thus the net coupling passes through zero. At larger offsets, the electric coupling changes its sign but remains smaller, since only the nearest halves of the SRRs are effectively interacting with this interaction decaying toward zero as the offset increases.

The magnetic interaction energy is also quite different for the two orientations with the magnetic interaction $\alpha_{L}$ calculated by Eq. (20) lying between the gap-to-gap and broadside curves. For the broadside-coupled case, the situation is qualitatively similar to the analytical result $\alpha_{L}$. At low offset, the magnetic field of one ring cuts through the other ring in the same direction to the surface normal, thus reinforcing the magnetic field and increasing the total energy. As the offset is increased, the situation gradually shifts to become like a pair of loops in the same plane, where the field from one ring cuts through the other in the opposite direction with respect to the surface normal. Hence, $\alpha_{\mathrm{bc}}$ undergoes a change in sign. However, in comparison to the ring with uniform current, the coupling is substantially more negative. This is due to the current maxima being on opposite sides of the rings, and hence further away from each other.

For the gap-to-gap orientation the magnetic interaction is much stronger for low $\delta a$. This is due to the current maxima being located near each other which produces a stronger contribution to the integral in $W_{H, 12}$ thus increasing $\alpha$. As the rings are further separated from each other, the interaction energy reduces, but does not undergo a change in sign. We can intuitively understand this by neglecting the small contributions of the current in the region near the gaps, thus we effectively have two linear current elements in the same plane which always interact with the same sign. However, this balance is not universal and is determined by the specific geometry and parameters. To check this, we studied a geometry with a very small gap so that the current distribution was 
(a) Broadside coupled orientation

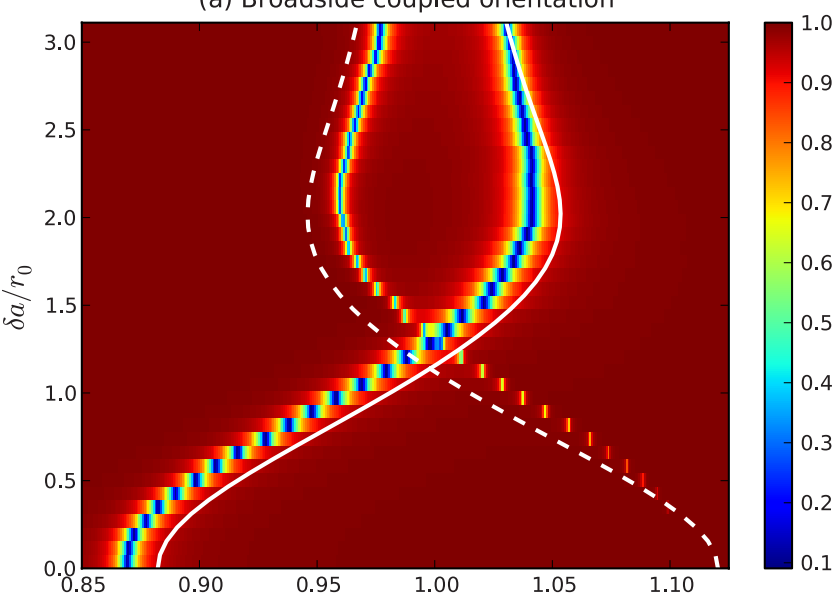

(b) Gap to gap orientation

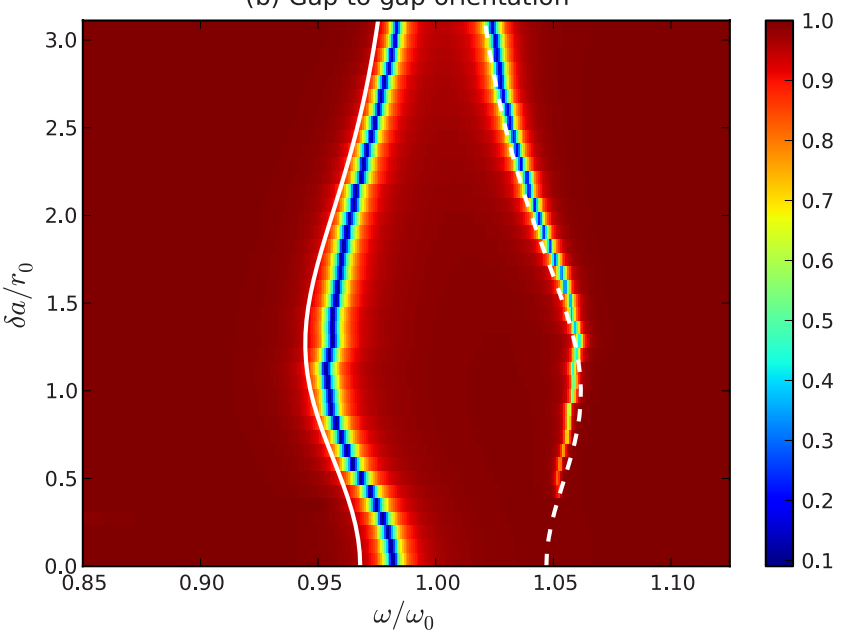

FIG. 5. (Color online) Numerical results. Transmission spectrum for a pair of (a) broadside-coupled and (b) gap-to-gap oriented rings. Solid line: $\omega_{S}$ from Eq. (13), broken line: $\omega_{A S}$ from Eq. (14)

much more homogeneous with lower resonance frequency. In this simulation (not shown) the magnetic coupling did change sign and both values of $\alpha$ converged closely to $\alpha_{L}$.

In order to verify that the calculated coupling correctly describes the frequency splitting of this system, we compare the frequency shift predicted by Eqs. (13) and (14) with that obtained from the full numeric simulations. For consistency with our interaction-energy approach we assume a homogeneous free-space background. We use the frequency domain solver of the commercial software package CST MICROWAVE STUDIO (Ref. 30) to model a pair of rings, in a unit cell with periodic boundary conditions in the directions transverse to the propagation direction. This periodic system enables us to define a transmission coefficient, and the boundaries are 10 $\mathrm{mm}$ from the rings. This value is chosen to be large enough so that there is no significant interaction with periodic neighbors, yet small enough to avoid significant scattering into higher order diffraction modes. Thus we can consider this the limiting case of a highly dilute metamaterial slab.

The transmission spectrum as a function of offset is plotted in Fig. 5. We see that all the important features of the mode splitting are represented correctly by our single-mode theory of coupled SRRs. Expansion of Eqs. (13) and (14) for small coupling predicts frequency splitting of $\Delta \omega= \pm(\beta$ $-\alpha) / 2$, hence the curves are approximately symmetric about $\omega_{0}$.

The strong splitting observed for the broadside-coupled orientation is due to the opposite signs of the electric and magnetic coupling. At $\delta a / r_{0} \approx 1.1$ both $\alpha_{\mathrm{bc}}$ and $\beta_{\mathrm{bc}}$ change signs, hence the crossing of the symmetric and antisymmetric modes is observed. In contrast, we see that for the gapto-gap orientation for small offset $\alpha_{\mathrm{gtg}}$ and $\beta_{\mathrm{gtg}}$ are of the same sign, and thus they have an opposing effect, resulting in small frequency splitting. Since $\beta$ decreases much faster and changes sign, the result in maximum frequency splitting for $\delta a / r_{0}$ between 1.1 and 1.5 . We note that in Ref. 12 the resonant frequency based on $\alpha_{L}$ was compared with experimental results for the broadside-coupled orientation and strong disagreement was found.

It can be seen that the calculated transmission through the cell exhibits different depths of the resonance for the symmetric and antisymmetric modes. This is due to the different efficiency of coupling between the modes and the incident plane wave. For instance, it is impossible to excite the antisymmetric mode with a normally incident plane wave for $\delta a=0$, since both rings are excited in phase. As the offset is increased, some retardation between the rings occurs and excitation of the antisymmetric mode is allowed.

There are several reasons for the small quantitative disagreement between the exact calculations and those based on the calculated interaction energy. First, the minimum of transmission occurs at a frequency slightly different from the resonant frequency, due to coupling effects (impedance matching) between the incident wave and the ring. Second, there may be some small contribution of higher SRR eigenmodes due to perturbation of the charge and current distributions. Third, there may still be some small influence of the periodic boundaries. Finally, our developed relations neglect retardation, which is strictly valid in the subwavelength limit, whereas the outer radius of the rings is $0.18 \lambda$ at $\omega_{0}$. Retardation has previously been shown to modify the interaction between SRRs through its influence on the dispersion of magnetoinductive waves in arrays. ${ }^{31-33}$

We emphasize that our approach developed in Sec. II is advantageous over the direct numerical calculation. First, once the mode profile is known, calculation of the frequencies in Fig. 5 takes approximately $30 \mathrm{~s}$ on a single CPU whereas the direct calculation of the full spectrum takes several hours on a multicore machine. Second, we are clearly able to demonstrate the nature of the coupling, which yields insight into the tuning behavior.

\section{TUNING INTERACTION IN A BULK METAMATERIAL}

We now apply our approach to explain experimental results for tuning the response of a slab of metamaterial. The metamaterial is fabricated using photolithography to etch copper tracks onto FR4 printed circuit board, using the same geometry as in our numerical simulation of a pair of rings. The fabricated sample has 30 layers, each with five rings in the propagation direction and is only one ring in height (i.e., 
(a) Broadside-coupled orientation

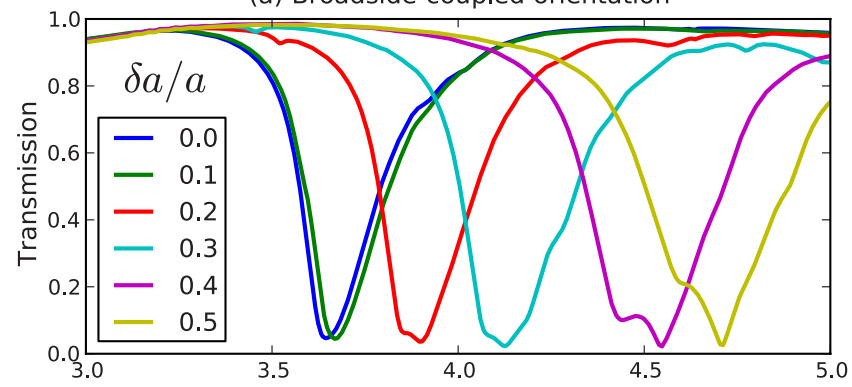

(b) Gap-to-gap orientation

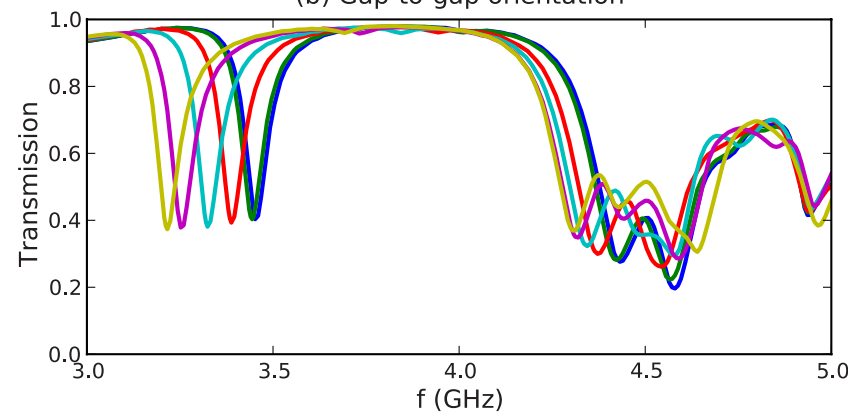

FIG. 6. (Color online) Experimental transmission while tuning $\delta a$ for split ring resonator slab in waveguide, for (a) broadsidecoupled and (b) gap-to-gap orientation of adjacent layers.

a $5 \times 30 \times 1$ array). The longitudinal period of the sample is $7 \mathrm{~mm}$, the transverse period is dictated by the sample thickness $(1.5 \mathrm{~mm})$ as there is no spacing between boards. As with the pair of rings, we have assembled slabs with two relative orientations of the split rings in adjacent planes-gap-to-gap and broadside-coupled. The sample is placed in the center of a WR229 rectangular metallic waveguide, with dimensions $58.17 \mathrm{~mm} \times 29.08 \mathrm{~mm}$, excited at its dominant $\mathrm{TE}_{10}$ mode. We removed the influence of the coaxial adapters and feeding waveguide sections by performing a through-reflectline calibration. ${ }^{34}$ In Fig. 6 we show the experimentally obtained transmission through each slab of closely coupled SRRs with the corresponding reflection shown in Fig. 7.

The shift of resonant frequency shows good qualitative agreement with the results for a pair of rings presented in Sec. III with very similar changes in the spectrum observed (noting that $\delta a / a=0.5$ corresponds to $\delta a / r_{0}=1.56$ ). However, for the gap-to-gap orientation, numerical simulation of a system of two boards with five rings each, and periodic boundaries in both transverse directions (not shown), is qualitatively similar to the experimentally observed results but quantitatively highly inaccurate. The reason turns out to be the loss of symmetry when the system is placed inside the waveguide because the upper and lower waveguide walls do not correspond to periodic boundaries but instead represent planes of mirror reflection. Therefore, this system must be described as having a superlattice arrangement in the vertical as well as horizontal planes with each supercell consisting of four SRRs. This cell has alternating orientation of the SRRs in the vertical direction corresponding to the planes of mirror symmetry, as shown in Fig. 8(b). Once this unit cell is taken into account, numerical simulations are in a good agreement with the experiment [Fig. 9(b)]. (a) Broadside-coupled orientation

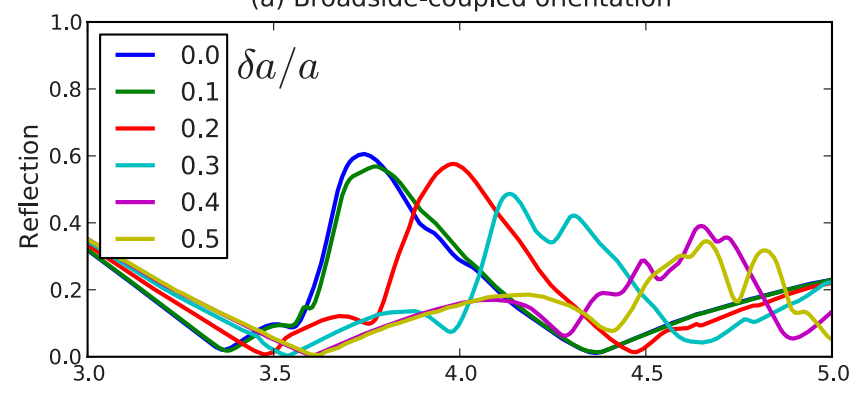

(b) Gap-to-gap orientation

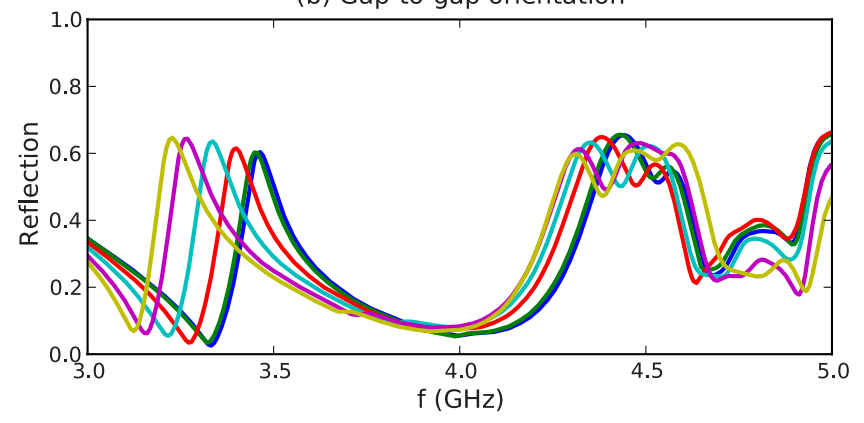

FIG. 7. (Color online) Experimental reflection while tuning $\delta a$ for split ring resonator slab in waveguide, for (a) broadside-coupled and (b) gap-to-gap orientation of adjacent layers.

Naturally, numerical simulations for the broadsidecoupled orientation also agree well with the experiment [Fig. 9(a)]. In this case a very similar result is provided with simple periodic boundary conditions (not shown). For this orientation the superlattice effectively formed by the waveguide shown in Fig. 8(a) does not have an essentially different symmetry to the original superlattice.

We can conclude that in both cases the dominant mode of the slab corresponds to the dominant symmetric mode of a pair of rings with a similar pattern of resonant frequency vs offset occurring. The weaker coupling to the modes for the gap-to-gap orientation is due to the shape of the symmetric mode. Its magnetic field has a large component parallel to that of the waveguide mode, however, its electric field is primarily longitudinal, in contrast to the transverse electric field of the waveguide mode.

In Fig. 9(b), we see two higher order modes, which most likely correspond to the higher order resonances observed in the experimental results in Fig. 6(b). From the numerical (a)

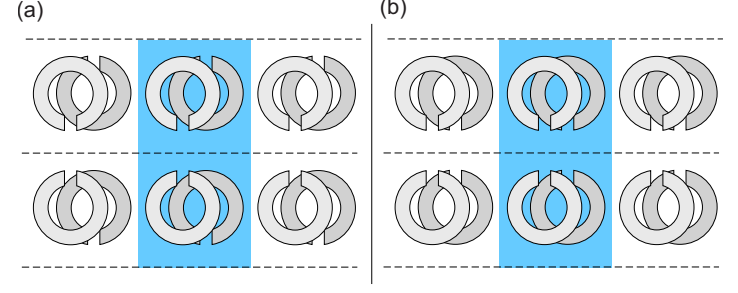

FIG. 8. (Color online) Schematic of the effective superlattice geometry corresponding to the waveguide measurement for (a) broadside-coupled and (b) gap-to-gap orientation. Dashed lines show planes of reflection symmetry and the shaded region shows the supercell with four SRRs. 

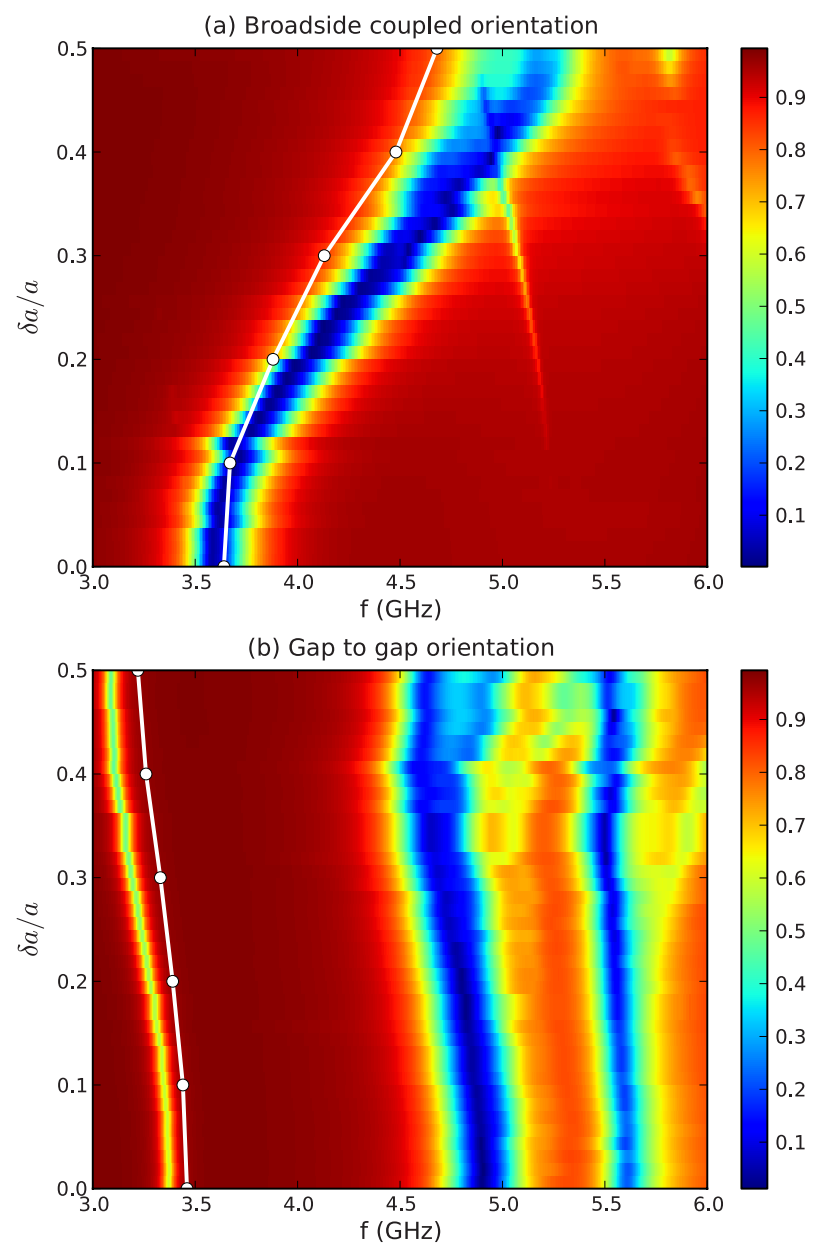

FIG. 9. (Color online) Numerically obtained transmission spectrum of metamaterial in waveguide, (a) broadside-coupled and (b) gap-to-gap orientation. The white line indicates experimentally obtained resonant frequencies.

simulations we observe that the current distributions of these modes are symmetric, thus they correspond to higher-order modes of the metamaterial slab and not to the antisymmetric mode of a pair of rings. In contrast, in Fig. 9(a) there is a weakly coupled antisymmetric mode, which we verified by inspection of the currents. This mode also qualitatively agrees with the corresponding mode of the pair of rings with somewhat weaker coupling due to the increased mismatch to the incident waveguide mode. This mode may correspond to some of the smaller features observable in Fig. 6(a), however, due to the size of these features this cannot be reliably determined.

We do not consider offsets greater than $0.5 a$, since in an infinite lattice only shifts between 0 and 0.5 are unique, while in a finite structure, larger shifts result in very irregular boundaries. Note that in the simulations we have neglected the effect of the mode profile of the rectangular waveguide, which would correspond to an effective variation in the angle of incidence of the plane wave as a function of frequency, which can result in a different response due to the anisotropy and non-negligible spatial dispersion of the medium. ${ }^{10}$

Clearly the coupling in the complete lattice is much more complicated than in the simple two-ring system, as the inter- actions between a large number of rings must be taken into account. In principle it is possible to extend the analysis of Sec. III to an arbitrary number of rings. However, the qualitative agreement between the experimental results and the modeled pair of rings suggests that the phenomenology developed for the two rings is generally applicable and leads to correct predictions.

Although an accurate generalization of our modeling approach to a bulk system lies beyond the scope of this paper, it is clear that the resulting homogenized effective metamaterial parameters will exhibit similar tuning pattern due to the resonance shift. Note that the introduction of the effective parameters is justified when the ratio of the unit-cell size to the incident wavelength is small. Therefore, when considering modifications of the lattice which create a superlattice structure, the size of the supercell should be smaller than the wavelength. There are homogenization approaches in the literature (e.g., Ref. 35) which include unknown parameters for interaction between resonant elements and our semianalytical approach would make an ideal tool for evaluating these constants.

\section{CONCLUSION}

We have analyzed the near-field coupling within metamaterials, considering both the relative orientation and the offset between the centers of two neighboring resonators. Using a pair of split ring resonators as a simple model, we have shown the coupling mechanisms at work in our recently proposed tuning scheme, based on the direct calculation of the interaction energy. We have confirmed that these mechanisms can predict qualitatively the performance of a realistic metamaterial structure. This paves a road toward a reliable design and development of tunable metamaterials for various applications.

We note that the specific geometry of the split rings can have a very significant influence on the qualitative nature of the coupling, including cases which run counter to our intuitive understanding of current loops interacting magnetically.

Finally, we point out that the approach developed here for modeling near-field effects is particularly promising for metamaterials scaled down to operate at optical frequencies. In the visible, the paradigm of ideally conducting metal fails and the area of applicability of circuit models is rather limited. In contrast, the consideration in terms of excitation and interaction of plasmonic standing waves will provide a clear physical picture.

\section{ACKNOWLEDGMENTS}

The authors are grateful to Lukas Jelinek and Ricardo Marqués (University of Seville) for helpful discussions. This work was supported by the Australian Research Council. M.L. acknowledges hospitality of Nonlinear Physics Center and support of the Spanish Junta de Andalusia under Project No. P06-TIC-01368. M.G. acknowledges support from the Russian Academy of Sciences, BPS Program "Physics of new materials and structures." 
*david.a.powell@anu.edu.au

${ }^{1}$ M. G. Silveirinha and C. A. Fernandes, Phys. Rev. B 78, 033108 (2008).

${ }^{2}$ R. W. Ziolkowski, Phys. Rev. E 70, 046608 (2004).

${ }^{3}$ J. Pendry, A. Holden, D. Robbins, and W. Stewart, IEEE Trans. Microwave Theory Tech. 47, 2075 (1999).

${ }^{4}$ M. Gorkunov and M. Lapine, Phys. Rev. B 70, 235109 (2004).

${ }^{5}$ I. Shadrivov, S. Morrison, and Y. Kivshar, Opt. Express 14, 9344 (2006).

${ }^{6}$ T. H. Hand and S. A. Cummer, J. Appl. Phys. 103, 066105 (2008).

${ }^{7}$ A. Degiron, J. Mock, and D. Smith, Opt. Express 15, 1115 (2007).

${ }^{8}$ M. Gorkunov, M. Lapine, E. Shamonina, and K. Ringhofer, Eur. Phys. J. B 28, 263 (2002).

${ }^{9}$ J. D. Baena, L. Jelinek, R. Marqués, and M. Silveirinha, Phys. Rev. A 78, 013842 (2008).

${ }^{10}$ C. R. Simovski, Metamaterials 2, 169 (2008).

${ }^{11}$ I. V. Shadrivov, D. A. Powell, S. K. Morrison, Y. S. Kivshar, and G. N. Milford, Appl. Phys. Lett. 90, 201919 (2007).

${ }^{12}$ M. Lapine, D. Powell, M. Gorkunov, I. Shadrivov, R. Marqués, and Y. Kivshar, Appl. Phys. Lett. 95, 084105 (2009).

${ }^{13}$ J. García-García, F. Martín, J. D. Baena, R. Marqués, and L. Jelinek, J. Appl. Phys. 98, 033103 (2005).

${ }^{14}$ P. Gay-Balmaz and O. J. F. Martin, J. Appl. Phys. 92, 2929 (2002).

${ }^{15}$ H. Liu, Y. M. Liu, T. Li, S. M. Wang, S. N. Zhu, and X. Zhang, Phys. Status Solidi B 246, 1397 (2009).

${ }^{16}$ T. Q. Li, H. Liu, T. Li, S. M. Wang, J. X. Cao, Z. H. Zhu, Z. G. Dong, S. N. Zhu, and X. Zhang, Phys. Rev. B 80, 115113 (2009).

${ }^{17}$ B. Kanté, A. de Lustrac, and J.-M. Lourtioz, Photonics Nanostruct. Fundam. Appl. 8, 112 (2010).

${ }^{18}$ J. Zhou, T. Koschny, M. Kafesaki, and C. M. Soukoulis, Phys. Rev. B 80, 035109 (2009).

${ }^{19}$ M. Decker, S. Linden, and M. Wegener, Opt. Lett. 34, 1579
(2009).

${ }^{20}$ R. Singh, C. Rockstuhl, F. Lederer, and W. Zhang, Phys. Rev. B 79, 085111 (2009).

${ }^{21}$ N. Liu, S. Kaiser, and H. Giessen, Adv. Mater. 20, 4521 (2008).

${ }^{22}$ O. Sydoruk, A. Radkovskaya, O. Zhuromskyy, E. Shamonina, M. Shamonin, C. J. Stevens, G. Faulkner, D. J. Edwards, and L. Solymar, Phys. Rev. B 73, 224406 (2006).

${ }^{23}$ H. Liu, D. A. Genov, D. M. Wu, Y. M. Liu, J. M. Steele, C. Sun, S. N. Zhu, and X. Zhang, Phys. Rev. Lett. 97, 243902 (2006).

${ }^{24}$ F. Hesmer, E. Tatartschuk, O. Zhuromskyy, A. A. Radkovskaya, M. Shamonin, T. Hao, C. J. Stevens, G. Faulkner, D. J. Edwards, and E. Shamonina, Phys. Status Solidi B 244, 1170 (2007).

${ }^{25}$ N. Liu, H. Liu, S. Zhu, and H. Giessen, Nat. Photonics 3, 157 (2009).

${ }^{26}$ P. Arcioni, M. Bressan, and L. Perregrini, IEEE Trans. Microwave Theory Tech. 45, 436 (1997).

${ }^{27}$ L. D. Landau and E. M. Lifschitz, Electrodynamics of Continuous Media (Pergamon Press, Oxford, 1984).

${ }^{28}$ R. Marqués, F. Mesa, J. Martel, and F. Medina, IEEE Trans. Antennas Propag. 51, 2572 (2003).

${ }^{29}$ M. Shamonin, E. Shamonina, V. Kalinin, and L. Solymar, Microwave Opt. Technol. Lett. 44, 133 (2005).

${ }^{30}$ Computer Simulation Technology, http://www.cst.com

${ }^{31}$ A. Radkovskaya, M. Shamonin, C. Stevens, G. Faulkner, D. Edwards, E. Shamonina, and L. Solymar, J. Magn. Magn. Mater. 300, 29 (2006).

${ }^{32}$ O. Zhuromskyy, O. Sydoruk, E. Shamonina, and L. Solymar, J. Appl. Phys. 106, 104908 (2009).

${ }^{33}$ M. Decker, S. Burger, S. Linden, and M. Wegener, Phys. Rev. B 80, 193102 (2009).

${ }^{34} \mathrm{G}$. Engen and C. Hoer, IEEE Trans. Microwave Theory Tech. 27, 987 (1979).

${ }^{35}$ J. Petschulat, C. Menzel, A. Chipouline, C. Rockstuhl, A. Tünnermann, F. Lederer, and T. Pertsch, Phys. Rev. A 78, 043811 (2008). 